\title{
AVALIAÇÃO DAS CONDUTAS DE PUNÇÃO E MANUTENÇÃO DO CATETER INTRAVENOSO PERIFÉRICO
}

\section{EVALUATION OF PERIPHERAL INTRAVENOUS CATETER PUNCTURE AND MAINTENANCE PROCEDURES}

\section{EVALUACIÓN DE LAS CONDUCTAS DE PUNCIÓN Y MANTENIMIENTO DEL CATETER INTRAVENOSO PERIFÉRICO}

Diego Alcântara Alves ${ }^{1}$, Thabata Coaglio Lucas ${ }^{2}$, Dulce Aparecida Martins ${ }^{3}$, Rayana Santos Cristianismo ${ }^{4}$, Emerson Vinícius de Oliveira Braga ${ }^{5}$, Helisamara Mota Guedes ${ }^{6}$.

\begin{abstract}
RESUMO
Objetivo: avaliar a equipe de enfermagem quanto às condutas de punção e manutenção do cateter intravenoso periférico. Método: estudo transversal, realizado entre os meses de setembro e dezembro de 2017, em uma instituição filantrópica no interior de Minas Gerais, nas clínicas cirúrgica, pediátrica e maternidade. Utilizou-se o índice de concordância de Kappa, não ponderado, com intervalo de confiança de $95 \%$ e nível de significância $p<0.05$. Resultados: a concordância entre os especialistas e a equipe de enfermagem, segundo o índice de Kappa, foi moderada $(0,41-0,60)(p<0,05)$. Sete pacientes $(1,8 \%)$ apresentaram eritema, com dor e/ou edema no local de inserção do cateter e foram classificados como grau 2. Observou-se que onze cateteres $(2,8 \%)$ estavam com o dispositivo destampado no momento da visita, 138 (35,5\%) com o curativo molhado, 206 (53\%) apresentavam sujidades no curativo e no extensor (dupla via), $117(30,1 \%)$ estavam sem data, $123(31,6 \%)$ sem a assinatura do profissional que realizou a punção. Conclusão: Apesar de ser um desafio para a prática clínica, seguir diretrizes baseadas em evidências científicas, este estudo contribui para evidenciar que, sem medidas de vigilância e treinamento da equipe multiprofissional, as medidas de controle de infecção, associadas ao cateter venoso periférico, podem não ser adotadas e refletir risco para o paciente.
\end{abstract}

Descritores: Flebite; Observação; Infecção; Enfermagem.

\section{ABSTRACT}

Objective: evaluate the nursing team regarding peripheral intravenous catheter puncture and maintenance. Method: a crosssectional study was carried out between September and December of 2017 in a philanthropic institution in the country-side of Minas Gerais, at the surgical, pediatric and maternity clinics. The unweighted Kappa agreement index with $95 \%$ confidence interval and a significance level $p<0.05$ were adopted. Results: the agreement between the specialists and the nursing team according to the Kappa index was moderate $(0.41-0.60)(p<0.05)$. Seven patients $(1.8 \%)$ presented erythema with pain and/or edema at the insertion site and were classified as grade 2 . It was observed that 11 catheters $(2.8 \%)$ had uncovered devices at the time of the visit, $138(35.5 \%)$ presented wet dressings, 206 (53\%) presented dirt in the dressing and in the extensor (double route), 117 (30.1\%) were undated, and $123(31.6 \%)$ had no signature of the professional who performed the puncture. Conclusion: although it is a challenge for clinical practice to follow guidelines based on scientific evidence, this study contributes to show that without surveillance measures and training of the multiprofessional team, control measures of infections associated with peripheral venous catheters may not be adopted and translate into risk to patients.

Descriptors: Phlebits; Observation; Infection; Nursing.

\section{RESUMEN}

Objetivo: evaluar el equipo de enfermería en cuanto a las conductas de punción y mantenimiento del catéter intravenoso periférico. Método: estudio transversal, realizado entre los meses de septiembre y diciembre de 2017, en una institución filantrópica en el interior de Minas Gerais, en las clínicas quirúrgica, pediátrica y maternidad. Se utilizó el índice de concordancia de Kappa no ponderado con intervalo de confianza del $95 \%$ y nivel de significancia $p<0.05$. Resultados: la concordancia entre los especialistas y el equipo de enfermería, según el índice de Kappa, fue moderada $(0,41-0,60)(p<0,05)$. Siete pacientes $(1,8 \%)$ presentaron eritema, con dolor y/o edema en el lugar de inserción del catéter y se clasificaron como grado 2 . Se observó que $11(2,8 \%)$ catéteres estaban con el dispositivo de estampado en el momento de la visita, 138 (35,5\%) con el vendaje mojado, 206 (53\%) presentaban suciedad en el vendaje y en el extensor (doble vía), 117 (30,1\%) estaban sin fecha, 123 (31,6\%) sin la firma del profesional que realizó la punción. Conclusión: A pesar de ser un desafío para la práctica clínica seguir directrices basadas en evidencias científicas, este estudio contribuye a evidenciar que, sin medidas de vigilancia y entrenamiento del equipo multiprofessional, las medidas de control de infección, asociadas al catéter venoso periférico, pueden no ser adoptadas y reflejar el riesgo para el paciente.

Descriptores: Flebitis; Observación; Infección; Enfermería.

${ }^{1}$ Graduado em Enfermagem pela Universidade Federal dos Vales do Jequitinhonha e Mucuri. ${ }^{2}$ Graduada em Enfermagem. Doutora em Engenharia Mecânica/ Bioengenharia pela Universidade Federal de Minas Gerais. Docente na Universidade Federal dos Vales do Jequitinhonha e Mucuri. ${ }^{3}$ Graduada em Enfermagem. Mestre em Enfermagem Universidade de São Paulo. Docente na Universidade Federal dos Vales do Jequitinhonha e Mucuri. ${ }^{4}$ Graduada em Enfermagem pela Universidade Federal dos Vales do Jequitinhonha e Mucuri. ${ }^{5}$ Graduado em Enfermagem pela Universidade Federal dos Vales do Jequitinhonha e Mucuri. ${ }^{6}$ Graduada em Enfermagem. Doutora em Enfermagem pela Universidade Federal de Minas Gerais. Docente na Universidade Federal dos Vales do Jequitinhonha e Mucuri. 


\section{INTRODUÇÃO}

A inserção do Cateter Intravenoso Periférico (CIP) é um dos métodos mais utilizados para terapia de infusão em pacientes hospitalizados ${ }^{(1-2)}$. As complicações como a infiltração, oclusão, mal funcionamento e flebite, no entanto, podem gerar aumento da morbidade, mortalidade, da permanência e custo na hospitalização(2).

Estudos brasileiros têm mostrado uma incidência de $22,9 \%$ de flebite pós-infusional e de $72,6 \%$ de flebite química ${ }^{(3-4)}$. Nos Estados Unidos, Inglaterra e Irã há uma prevalência de flebite pósinfusional de 20 a $80 \%{ }^{(5)}$. Conforme dados da Nursing Society of Intravenous Fluid, a taxa aceitável de flebite, em uma determinada população de pacientes, é de $5 \%$ ou menos ${ }^{(5)}$.

O acesso venoso através CIP é um procedimento invasivo em que ocorre a instalação de um dispositivo estéril no interior do vaso para terapia intravenosa. Sendo assim, pode ser considerado um procedimento crítico e invasivo, visto que o cateter rompe a pele que confere proteção natural e adentra no interior da veia. Tal técnica, se não atendidos os requisitos de controle de infecção e adequação da habilidade durante o procedimento, pode levar a uma inflamação na parede do vaso sanguíneo ${ }^{(6)}$.

Nesse sentido, a flebite, inflamação das células endoteliais da parede venosa, relacionada ao uso do CIP, é considerada um dos eventos adversos mais frequentes na prática diária das instituições hospitalares. A prevenção da mesma ainda é uma lacuna no conhecimento científico, uma vez que pode envolver fatores mecânicos e químicos ${ }^{(2,7-8)}$.

Cateteres com menor calibre podem causar menos flebite mecânica à parede da veia pela cânula e menor obstrução do fluxo sanguíneo dentro do vaso quando comparados àqueles de maior calibre ${ }^{(7-10)}$. Por outro lado, são necessários cuidados, sobretudo na administração de medicamentos, que deve ser lenta, para possibilitar um adequado fluxo sanguíneo no interior do vaso de forma que não irrite a parede do vaso levando a uma possível flebite química.

As manifestações da flebite são caracterizadas por dor, edema, hiperemia no local e endurecimento ${ }^{(9-10)}$. Em casos de evoluções, pode surgir cordão fibroso palpável, com presença ou não de secreção purulenta no sítio de inserção do cateter ${ }^{(8-9)}$. A flebite pósinfusional pode ocorrer após o encerramento da infusão e da retirada do cateter, normalmente identificada dentro de 48 horas após a remoção do $\mathrm{CIP}^{(9-12)}$. A manutenção do CIP requer avaliação a cada quatro horas ou de acordo com a criticidade do paciente, buscando identificar sinais clínicos sugestivos de flebite ${ }^{(13)}$.

Segundo a Agência Nacional da Vigilância Sanitária (2017), ações como higiene das mãos, escolha de veias mais calibrosas, escolha do menor dispositivo indicado à infusão, fixação adequada para prevenir irritação mecânica, punções realizadas por profissionais tecnicamente habilitados e que tenham conhecimento suficiente para tal, serão suficientes para reduzir as complicações relacionadas ao $\mathrm{CIP}^{(13)}$. O rodízio do local do acesso a cada 96 horas é indicado, porém, em se tratando de pacientes pediátricos ou com difícil acesso, essa recomendação deve ser reconsiderada ${ }^{(13)}$.

Condutas de punção e manutenção do sítio de inserção como oclusão e fixação do curativo, aspecto limpo e seco devem ser observados diariamente pela equipe de enfermagem, pois uma preservação inadequada do sítio de inserção pode levar a eventos adversos nos pacientes. Estudo de intervenção mostrou que a fixação inadequada do CIP levou a $71 \%$ de incidência de flebite $^{(2)}$. A fixação deve ser realizada com material estéril e transparente, de modo a favorecer a inspeção do sítio de inserção ${ }^{(13)}$.

Apesar da importância, estudos de abordagem para o curativo e fixação são ainda insuficientes e recebem pouca atenção na comunidade científica ${ }^{(2)}$. A fixação suja e úmida pode levar a formação de biofilmes e proliferação de bactérias no sítio de inserção e até mesmo no interior do lúmen do cateter ${ }^{(2,7-8)}$. Os conectores ou tampas dos cateteres também podem ser contaminados por bactérias de forma cruzada quando medidas de assepsia não são realizadas de forma adequada. Destaca-se aqui que, apesar de os conectores serem recomendados como uso único, o elevado custo para troca de novos conectores faz com que a maioria das instituições não os descarte e siga recomendações padronizadas de friç̧ão alcoólica após manuseio $^{(2,13)}$.

As punções por CIP devem ser minimizadas ao considerar o mesmo paciente, evitando a troca programada do cateter, a qual não reduz os riscos de flebite e outras complicações relacionadas a esse instrumento; antes, causa aumento do desconforto em pacientes e carga de trabalho em enfermeiros ${ }^{(5-8)}$. 
Estudos indicam que, para evitar complicações associadas ao CIP, como a flebite, educação continuada em habilidade e técnicas de inserção contribuem para reduzir as dificuldades de inserção e manutenção do $\operatorname{CIP}^{(2,5,7-9)}$. Para a equipe de enfermagem, no entanto, ainda é um desafio a aplicação de técnicas adequadas nas atividades práticas e clínicas nas instituições hospitalares. Diante do exposto, o estudo tem, como objetivo, avaliar a equipe de enfermagem quanto às condutas de punção e manutenção do cateter intravenoso periférico.

\section{MÉTODO}

Estudo transversal, realizado com a equipe de enfermagem de uma instituição filantrópica no interior de Minas Gerais. O período de estudo foi entre os meses de setembro e dezembro de 2017. A instituição possui uma equipe de 44 profissionais compondo a equipe de enfermagem, distribuídos entre as clínicas cirúrgica, maternidade e pediatria, sendo um total de 36 técnicos de enfermagem e 8 enfermeiros. A amostra do estudo incluiu 32 profissionais da equipe de enfermagem. Houve uma perda de $27,3 \%$ devido a 3 profissionais se encontrarem em período de férias, 1 ter sido remanejado da equipe e 8 terem se recusado a participar da pesquisa.

Para a coleta de dados aplicou-se um questionário validado ${ }^{(14)}$, contendo 12 questões com as seguintes variáveis: sexo, idade, indicação do CIP, modo de preparo da medicação e eventos adversos associados a ela, seleção de materiais para punção venosa, técnica de retirada e inserção do CIP e antissepsia da pele. Cada questão possuía cinco alternativas de respostas.

Realizou-se também a técnica da observação direta por um período de 4 meses do CVP dos pacientes internados nas clínicas cirúrgica, pediátrica e na maternidade. Os dados foram coletados a partir de visitas diárias, realizadas pelos pesquisadores, previamente treinados com condutas padronizadas. Foram realizadas 389 observações, sendo que o mesmo paciente pode ter sido observado mais de uma vez, quanto às seguintes variáveis categorizadas como "sim" ou "não": dispositivo tampado, seco, limpo, datado e assinado. Incluiu-se ainda a observação do conector do CIP a fim de se verificar se foi armazenada em local adequado ou não. Para este estudo, considerou-se adequado se o conector estava separado por paciente em um recipiente com tampa e seco, uma vez que a instituição ainda não adotou as recomendações da Anvisa sobre o descarte das tampas a cada uso ${ }^{(13)}$.

Foi avaliado o local de inserção do CIP quanto aos sinais flogísticos de flebite utilizando a escala de classificação proposta pela Infusion Nursing Society (INS) ${ }^{(1,3)}$. As categorias avaliadas foram: Grau 0 quando há ausência de sinais clínicos de flebite; Grau 1 quando há presença de eritema, com ou sem dor local; no Grau 2 há presença de eritema, com dor local e ou edema; Grau 3 caracterizado pela presença de eritema, dor local e/ou edema, com endurecimento e cordão fibroso palpável; no Grau 4 há presença de dor com eritema e/ou edema, com endurecimento e cordão fibroso palpável maior que $2,5 \mathrm{~cm}$ de comprimento e drenagem purulenta ${ }^{(1,3)}$.

A pesquisa foi aprovada sob o número de parecer 2.253.693 pelo Comitê de Ética em Pesquisa da Universidade Federal dos Vales do Jequitinhonha e Mucuri - UFVJM no ano de 2017.

Para análise dos dados, utilizou-se Statistical Package for the Social Sciences For Windows (SPSS, Inc., Chicago, IL, EUA), versão 20.0, para análise descritiva (Média (M) \pm Desvio Padrão (DP)) e de confiabilidade do questionário. A confiabilidade das respostas foi realizada por meio de análises entre as respostas de três especialistas no assunto (padrão ouro) confrontados com os resultados apresentados pelos sujeitos observados da equipe de enfermagem. Utilizou-se o índice de concordância de Kappa não ponderado com intervalo de confiança de 95\%. Adotou-se nível de significância $p<0,05$. O Kappa foi considerado adequado quando $>0,70$. Os valores de Kappa variam de -1 (ausência total de concordância) a 1 (concordância total).

A interpretação convencional dos valores Kappa é: 0,00-0,20 = concordância pobre; 0,21$0,40=$ regular $; 0,41-0,60=$ moderada $; 0,61-0,80=$ boa; $0,81-1,00=$ muito boa .

\section{RESULTADOS E DISCUSSÃO}

Dos 32 profissionais de enfermagem que participaram desta pesquisa, $7(21,9 \%)$ eram enfermeiros e 25 (78,1\%) técnicos de enfermagem, $87 \%$ eram do sexo feminino, média de idade de $32 \pm 7,61$ anos, sendo a idade mínima 20 anos e a máxima de 54 anos.

Quanto ao grau de concordância entre os especialistas e a equipe de enfermagem, a Tabela 1 apresenta os valores do índice de concordância de Kappa. 
Tabela 1 - Distribuição do grau de concordância entre os especialistas e a equipe de enfermagem segundo o índice de Kappa. Brasil, Diamantina, MG,2017.

\begin{tabular}{|c|c|c|c|}
\hline Questão & Resposta correta & $\begin{array}{l}\text { Índice de Kappa } \\
\text { (Erro padrão) }\end{array}$ & $\begin{array}{l}\text { IC } 95 \% \\
\text { Valor } p\end{array}$ \\
\hline 1 & Introdução de grande quantidade de líquido = venóclise. & $0,53(0,021)$ & $\begin{array}{l}-0,23-1,00 \\
p^{*}=0,001\end{array}$ \\
\hline 2 & $\begin{array}{l}\text { Administração de medicamento endovenoso }=\text { Não } \\
\text { executar a prescrição medicamentosa e terapêutica em } \\
\text { caso de identificação de erro ou ilegibilidade. }\end{array}$ & $0,48(0,023)$ & $\begin{array}{l}-0,32-1,00 \\
p^{*}=0,013\end{array}$ \\
\hline 3 & $\begin{array}{l}\text { Seleção de materiais para punção venosa periférica = } \\
\text { Cuba rim, luva de procedimento, seringa, agulha para } \\
\text { aspirar medicamento, medicamento a ser aspirado, } \\
\text { cateter periférico, garrote, esparadrapo, algodão e álcool } \\
\text { a } 70 \% \text {. }\end{array}$ & $0,52(0,023)$ & $\begin{array}{l}-0,12-1,00 \\
p^{*}=0,012\end{array}$ \\
\hline 4 & $\begin{array}{l}\text { Antissepsia da pele = no sentido do retorno venoso, } \\
\text { trocando as faces do algodão. }\end{array}$ & $0,41(0,038)$ & $\begin{array}{l}-0,26-1,00 \\
p^{*}=0,001\end{array}$ \\
\hline 5 & $\begin{array}{l}\text { Na punção venosa periférica = Selecionar o calibre da } \\
\text { agulha de acordo com a veia a ser puncionada. }\end{array}$ & $0,61(0,021)$ & $\begin{array}{l}-0,09-1,00 \\
p^{*}=0,002\end{array}$ \\
\hline 6 & $\begin{array}{l}\text { Ângulo de perfuração da punção venosa periférica }=5^{0} \text { a } \\
30^{\circ} \text { dependendo da profundidade do vaso. }\end{array}$ & $0,48(0,023)$ & $\begin{array}{l}-0,32-1,00 \\
p^{*}=0,001\end{array}$ \\
\hline 7 & $\begin{array}{l}\text { Bisel da agulha na punção venosa periférica = voltado } \\
\text { para cima. }\end{array}$ & $0,75(0,024)$ & $\begin{array}{l}0,43-1,00 \\
p^{*}=0,002\end{array}$ \\
\hline 8 & $\begin{array}{l}\text { Na punção venosa periférica com cateter agulhado = } \\
\text { inserir uma parte do cateter, aguardar o aparecimento de } \\
\text { sangue e deslizar o restante do cateter dentro da veia. }\end{array}$ & $0,43(0,024)$ & $\begin{array}{l}-0,21-1,00 \\
p^{*}=0,001\end{array}$ \\
\hline 9 & $\begin{array}{l}\text { Na punção venosa periférica com cateter agulhado, o } \\
\text { garrote }=\text { deve ser mantido durante a punção na veia. }\end{array}$ & $0,77(0,022)$ & $\begin{array}{l}0,45-1,00 \\
p^{*}=0,011\end{array}$ \\
\hline 10 & $\begin{array}{l}\text { Fixação do cateter: de acordo com o dispositivo a ser } \\
\text { utilizado. }\end{array}$ & $0,75(0,021)$ & $\begin{array}{l}0,43-1,00 \\
p^{*}=0,001\end{array}$ \\
\hline 11 & $\begin{array}{l}\text { Administração de medicamento via endovenosa = fazê-lo } \\
\text { lentamente. }\end{array}$ & $0,55(0,021)$ & $\begin{array}{l}-0,14-1,00 \\
p^{*}=0,001\end{array}$ \\
\hline 12 & $\begin{array}{l}\text { Na retirada do cateter agulhado = pressionar o local da } \\
\text { punção com o intuito de conter possíveis sangramentos e } \\
\text { a formação de hematomas. }\end{array}$ & $0,61(0,023)$ & $\begin{array}{l}-0,09-1,00 \\
p^{*}=0,001\end{array}$ \\
\hline
\end{tabular}

Fonte: Elaborada pelos próprios autores.

$*_{p}<0,05$.

O presente estudo mensurou o nível de conhecimento dos profissionais de enfermagem quando avaliado o índice de Kappa, que apesar de a maioria das perguntas avaliadas apresentarem valores $<0,70$, todas as variáveis foram significativas $(p<0,05)$. Tal fato pode demonstrar a insegurança da equipe de enfermagem, sobretudo, no conhecimento adequado da técnica de inserção do CIP agulhado como, por exemplo, o ângulo adequado de punção ou sentido da antissepsia da pele do paciente. Como verificado na Tabela 1 , a concordância para essa questão foi "moderada".
Um profissional com conhecimento adequado das técnicas de inserção e manutenção do CIP é capaz de colocar em prática sua competência clínica para aplicar medidas de prevenção de eventos adversos como a infiltração, extravasamento ou a flebite nas fases de pré-punção, punção e pós-punção ${ }^{(7-9,12)}$.

No que se refere às condutas de punção e manutenção encontradas no curativo do $\mathrm{CIP}$, a Tabela 2 apresenta a análise descritiva das variáveis analisadas na fase de observação direta. 
Tabela 2 - Distribuição das variáveis relacionadas às condutas de punção e manutenção do curativo no local de inserção do CIP, se seco, tampado, limpo, datado e assinado na fase de observação direta. Brasil, Diamantina, MG, 2017 ( $n=389$ ).

\begin{tabular}{lcccc}
\hline Condutas de punção e manutenção do curativo do CIP (n=389) & Sim & & Não & f \\
& f & \% & 11 & 2,8 \\
Ocluído & 378 & 97,2 & 138 & 35,5 \\
Seco & 251 & 64,5 & 53 & 30,1 \\
Limpo & 183 & 47 & 206 & 117 \\
Datado & 272 & 69,9 & 123 & 31,6 \\
Assinado & 266 & 68,4 & 270 & 69,4 \\
Conector em local inapropriado & 119 & 30,6 & 270 \\
\hline
\end{tabular}

Fonte: Elaborada pelos próprios autores.

Observou-se que 11 cateteres (2,8\%) estavam com o dispositivo destampado no momento da visita, 138 (35,5\%) com o curativo molhado, 206 (53\%) apresentavam sujidades no curativo e no extensor (dupla via), como sangue e medicação, $117(30,1 \%)$ estavam sem data, $123(31,6 \%)$ sem a assinatura do profissional que realizou a punção venosa periférica (Tabela 2).

Verificou-se que, 119 (30,6\%) dos pacientes tinham os conectores em locais inapropriados, como: pregadas no equipo e suporte de soro, na mesinha de cabeceira do paciente, dentro de um recipiente juntamente com conectores de diferentes pacientes e no bolso do jaleco do profissional.

Os outros $270(68,4 \%)$, estavam com o dispositivo, porém não estavam recebendo nenhuma solução por via endovenosa no momento da fase de observação direta (Tabela 2).

Quanto às condutas de punção e manutenção observadas na região de instalação do $\mathrm{CIP}$, verificou-se que $35,5 \%$ dos curativos não estavam secos e essa realidade é constante na instituição pesquisada (Tabela 2). Tal fato justifica-se devido à frequente umidade das fixações que pode estar associada ao número de infusões ou medicamentos por dia, a infiltrações e lavagens intralumens do equipo de infusão, além do próprio manuseio das fixações e cateteres $^{(9,15-17)}$.

Um estudo prospectivo de intervenção, realizado na Austrália, mostrou que metade dos curativos feitos em CIP não eram datados e nem assinados na instituição de estudo ${ }^{(18)}$. No entanto, pós intervenção educacional, datas e assinaturas nos curativos aumentaram significativamente de $36,4 \%$ para $50,0 \% \quad(p<0,05)^{(18)}$. No presente estudo, verificou-se que curativos não datados e assinados também constituíam um problema. Importante destacar que qualquer técnica que envolve o paciente deve ser bem documentada para futuras notificações de eventos adversos associadas ao CIP.

Para conscientizar a equipe de enfermagem, medidas educativas como monitorações ativas podem contribuir para melhorar o conhecimento da técnica de inserção e manutenção do $\operatorname{CIP}^{(15-16,18)}$. Estudos têm demonstrado uma redução nas taxas de complicações associadas ao CIP com abordagens agrupadas, incluindo uso de módulos de educação online, implementação de práticas baseadas em evidências de inserção e manutenção desses dispositivos. Adiciona-se a observação direta e monitorada dos profissionais de forma a obter um feedback da equipe ${ }^{(7-9,15-16)}$

Um estudo prospectivo realizado em Israel mostrou que houve diminuição da morbidade e no tempo de internação dos pacientes ao comparar a presença ou não de conectores de $\mathrm{CIP}^{(19)}$.

Uma análise multivariada foi utilizada nesse estudo prospectivo, incluindo variáveis como idade, sexo, tipo de curativo e conectores. Encontrou-se que somente a variável "conector" foi responsável pela redução da taxa de flebite $(p<0,01)$ na instituição ${ }^{(19)}$. Tal taxa poderia ser amenizada com a friç̧ão de antisséptico apropriado como o álcool a $70 \%$ para prevenção de infecções associadas aos conectores no momento de manuseá-los ${ }^{(12-13)}$.

A Tabela 3 apresenta a evolução da flebite de acordo com observações na região de punção e proximal do CIP. 
Tabela 3 - Evolução da flebite de acordo com observações da região de punção e proximal do CIP conforme o Infusion Nursing Society. Brasil, Diamantina, MG, 2017.

\begin{tabular}{lcc}
\hline Classificação & F & $\%$ \\
Grau O & 369 & 93,4 \\
Grau I & 12 & 3 \\
Grau II & 7 & 1,8 \\
Grau III & 1 & 0,3 \\
Grau IV & 0 & 0 \\
Grau V & 0 & 0 \\
\hline
\end{tabular}

Fonte: Elaborada pelos próprios autores.

Observou-se que 12 (3\%) dos pacientes apresentaram sinais clínicos como presença de eritema, com ou sem dor local, sendo classificado como grau 1; Sete $(1,8 \%)$ apresentaram eritema com dor e/ou edema no local de inserção do cateter e foram classificados como grau 2; apenas $1(0,3 \%)$ foi classificado como grau 3 , visto que apresentou eritema, dor local e/ou edema com endurecimento e cordão fibroso palpável.

Conhecer os possíveis eventos adversos que o paciente pode sofrer contribui para uma inserção do CIP bem sucedida ${ }^{(2,10-12)}$. Durante a punção venosa, por exemplo, diversos números de tentativas de inserção nos membros superiores podem desencadear uma flebite mecânica, muitas vezes, de difícil notificação ${ }^{(2,15)}$. Além disso, a flebite mecânica pode desenvolver com a movimentação inadequada dos membros superiores de forma que o cateter faça tensões de cisalhamento na camada íntima do vaso, isto é, força tangencial na parede da veia.

Um estudo prospectivo de coorte, realizado na Austrália verificou que a flebite mecânica foi associada ao lado dominante dos pacientes (1,39; IC 95\%, 1,09-1,77), isto é, o lado em que eles mais se movimentam ${ }^{(16)}$.

Para evitar a movimentação do cateter, ele deve ser estabilizado. Isto quer dizer preservar a integridade do acesso e prevenir o deslocamento ou perda do mesmo, seguindo técnicas assépticas $^{(2,16)}$. Vale lembrar que fitas adesivas não estéreis do tipo esparadrapo e fita microporada não devem ser utilizadas para estabilizar a CIP ou cobrir o cateter; a estabilização deve ser feita com dispositivo adesivo específico para tal, sendo transparente e estéril $^{(2,16)}$. Os sinais de flebite foram observados ao redor do curativo, geralmente com esparadrapo ou fita microporada.

$\mathrm{Na}$ instituição onde foi desenvolvido este estudo, a prática clínica ainda não contempla essa conduta, o que pode estar contribuindo para a alta incidência de flebites, uma vez que a não aplicação, no local de inserção do CIP, de fitas ou curativos estéreis, garante uma limitação no potencial de colonização de bactérias nessa região. O desenvolvimento da flebite, no entanto, depende de vários fatores como a osmolaridade e $\mathrm{pH}$ da solução injetada, sexo e idade do paciente, tempo de permanência do CIP, técnica utilizada para inserção desse cateter, sua fixação e localização, como também o material e o calibre do cateter escolhido ${ }^{(2,9,16)}$.

Outro fator que pode desencadear a flebite é o calibre da agulha no momento da punção venosa ${ }^{(15-17)}$. Estudo de meta-análise realizado em Taiwan mostrou que, quanto maior o calibre da agulha, maior a tendência a desenvolver flebite 0,88 (IC 95\%, 0,67-1,17) ${ }^{(17)}$. Cabe ressaltar que a seleção do cateter para inserção vai depender desse sítio e da solução que será infundida ${ }^{(16-17)}$.

Cabe à equipe de enfermagem reconhecer os sinais e sintomas da flebite a fim de notificar e tratar, o mais brevemente possível, o paciente. A flebite pode estreitar o lúmen das veias devido à hiperplasia luminal, diminuir o fluxo sanguíneo e desencadear um eritema e dor na região de punção ${ }^{(8,16-19)}$. Tais sinais e sintomas deveriam ser rapidamente identificados para que não ocorra a evolução da flebite, bem como a adoção de protocolos para a técnica e a educação continuada da equipe.

Um estudo prospectivo de coorte, realizado na Austrália, verificou associação significativa $(2,16 ; 95 \% \mathrm{Cl}, 1,26-3,71)$ entre a presença de hematoma no local de inserção e a flebite $^{(18)}$. Conhecendo esses sinais iniciais da flebite, a equipe deverá ser orientada no sentido de aumentar as medidas de controle durante a inserção e a manutenção do CIP, de modo a evitar maiores danos aos pacientes, assim como remoções desnecessárias do dispositivo, diminuição do custo com a saúde e risco de infecção, infiltração e inflamação do sítio de inserção ${ }^{(15-19)}$. 
Uma limitação do presente estudo foi a ausência de uma intervenção educativa com a equipe de enfermagem e uma comparação pré e pós intervenção associadas a taxas de incidência de flebite na instituição. Os resultados deste estudo foram apresentados à instituição pesquisada, apontando para a necessidade de se implantar medidas educativas e de vigilância ativa junto à equipe de enfermagem e à Comissão de Controle de Infecção Hospitalar (CClH), com revisão e adequação do protocolo de inserção e manutenção de CIP da instituição.

\section{CONCLUSÃO}

Os resultados deste estudo apontaram concordâncias variáveis (de "moderada" a "boa") no conhecimento da equipe de enfermagem sobre a inserção e manutenção do CIP. Tais resultados demonstraram um déficit no conhecimento técnico-científico na prática diária desses profissionais no que se refere ao procedimento de punção venosa periférica e instalação de cateter venoso.

Além disso, a observação das inadequações das condutas de punção e manutenção do curativo no local de instalação do cateter, como presença de umidade e não oclusão da fixação, aponta uma limitação da equipe nos cuidados de prevenção de infecção e inflamação na região de inserção do CIP. Isso mostra a necessidade da divulgação dos protocolos institucionais e a importância da educação continuada, a fim de evitar punções repetitivas, danos vasculares e consequente desconforto para o paciente.

Cabe ao enfermeiro responsável pelo setor, revisar os protocolos de inserção e de manutenção do CIP, com especial atenção ao controle dos fatores predisponentes para a infecção, tais como data de vencimento, fixação dos curativos, proteção dos CIP durante o banho, escolha de calibres menores para a punção, dentre outros propostos pela Anvisa.

Ainda é um desafio para a prática clínica seguir diretrizes baseadas em evidências científicas, uma vez que as mudanças nos protocolos institucionais exigem aprovação dos administradores por envolver custos com equipamentos para melhorar a conformidade das intervenções. No entanto, as variáveis avaliadas neste estudo, associada à técnica de inserção e manutenção do CIP são de fácil implementação quando associadas às medidas de vigilância institucionais.
Sendo assim, este estudo contribui para o desenvolvimento de futuras pesquisas de intervenção e experimentais, para que lacunas e evidências científicas sejam ampliadas e divulgadas, não somente para a equipe de enfermagem, mas para as instituições de saúde, com o envolvimento da $\mathrm{CClH}$, do setor de compras e a padronização de processos.

\section{REFERÊNCIAS}

1 - Mattox EA. Complications of peripheral venous access devices: Prevention, detection, and recovery strategies. Crit Care Nurs2 2017;37(2): e1-e14. DOI: $10.4037 / \mathrm{ccn} 2017657$

2 - Rickard CM, Marsh NM, Webster J, Playford G, McGrail MR, Larsen $E$, et al. Securing all intraVenous devices effectively in hospitalised patients - the SAVE trial: Study protocol for a multicentre randomised controlled trial. BMJ Open 2015;5(9):1-7. DOI: 10.1136/bmjopen2015-008689

3 - Urbanetto JS, Muniz FOM, Silva RM, Freitas APC, Oliveira APR, Santos JCR. Incidência de flebite e flebite pós-infusional em adultos hospitalizados. Rev Gaúcha Enferm. 2017;38(2):110. DOI: $10.1590 / 1983-1447.2017 .02 .58793$

4 - Beccaria LM, Contrin LM, Werneck AL, Machado BD, Sanches EB. Incidência de flebites em pacientes adultos. Rev Enferm UFPE 2018;12(3):745-52. DOI: 10.5205/1981-8963v12i3a230454p745-752-2018

5 - Badadi ME, Ghadiriyan R, Hosseini SM. The effect of saline lock on phlebitis rates of patients in cardiac care units. Iran J Nurs Midwifery Res. 2015;20(4):496-501. DOI: 10.4103/ 17359066.161006

6 - Pereira KC, Cogo ALP, Silva APSS. Análise crítica dos vídeos sobre punção venosa periférica com cateter disponibilizados no Youtube. Rev Min Enferm. 2016;20(970):1-6. DOI: 10.5935/1415$\underline{2762.20160040}$

7 - Xu L, Hu Y, Huabg X, Fu J, Zhang J. Clinically indicated replacement versus routine replacement of peripheral venous catheters in adults: A nonblinded, clusterrandomized trial in China. Int J Nurs Pract. 2017;23(6):1-45. DOI: 10.1111/ijn.12595

8 - Parker SI, Benzies KM, Hayden KA, Lang ES. Effectiveness of interventions for adult peripheral intravenous catheterization: A systematic review and meta-analysis of randomized controlled trials. Int Emerg Nurs. 2017;31:15-21. DOI: 10.1016/j.ienj.2016.05.004 
9 - Li X-F, Liu W, Qin Y. Nurses' perception of risk factors for infusion phlebitis: A cross-sectional survey. Chinese Nursing Research 2016;3:37-40. DOI: 10.1016/j.cnre.2016.03.002

10 - Milutinović $D, \operatorname{Simin} D$, Zec D. Fatores de risco para flebite: estudo com questionário sobre a percepção dos enfermeiros. Rev Latino-Am Enfermagem 2015;23(4):677-84. DOI: 10.1590/0104-1169.0192.2603

11 - O'Grady NP, Alexander M, Burns LA, Dellinger EP, Garland J, Heard SO, et al. Guidelines for the prevention on of intravascular catheter-related infeccions. Clin Infect Dis. 2011;52(9):162-93. DOI: $10.1093 / \mathrm{cid} / \mathrm{cir} 257$

12 - Tertuliano AC, Borges JLS, Fortunato RAS, Oliveira AL, Poveda VB. Flebite em acessos venosos periféricos de pacientes de um hospital do Vale do Paraíba. Rev Min Enferm. 2014; 8(2):334-9. DOI: 10.5935/1415-2762.20140026 13 - Agência Nacional de Vigilância Sanitária. Medidas de Prevenção de Infecção Relacionada à Assistência à Saúde. Brasília: Anvisa; 2017.

14 - Oliveira AKA. Validação de instrumentos para avaliação do conhecimento e da habilidade acerca da punção venosa periférica com cateter agulhado [tese]. Natal: Universidade Federal do Rio Grande do Norte; 2013.

15 - Murayama R, Uchida H, Oe H, Takahashi T, Oya H, Komiyama C, Sanada H. Removal of peripheral intravenous catheters due to catheter failures among adult patients. J Infus Nurs. 2017; 40(4):224-31.

DOI:

\subsection{7/NAN.0000000000000168}

16 - Marsh N, Webster J, Larson E, Cooke M, Mihala $\mathrm{G}$, Rickard CM. Observational study of peripheral intravenous catheter outcomes in adult hospitalized patients: A multivariable analysis of peripheral intravenous catheter failure. J Hosp Med. 2018;13(2):83-9. DOI: 10.12788/jhm.2867

17 - Chang WP, Peng YX. Occurrence of phlebitis: A systematic review and meta-analysis. Nurs Res. 2018;67(3):252-60.

DOI:

\subsection{7/NNR.0000000000000279}

18 - Yagnik L, Graves A, Thong K. Plastic in patient study: Prospective audit of adherence to peripheral intravenous cannula monitoring and documentation guidelines, with the aim of reducing future rates of intravenous cannularelated complications. Am J Infect Control. 2017;45(1):34-8. DOI: 10.1016/j.ajic.2016.09.008 19 - Ronen 0, Shiomo F, Ben-Adiva G, Edri Z, Shema-Didi L. A prospective clinical trial to assess peripheral venous catheter-related phlebitis using needleless connectors in a surgery department. Am J Infect Control. 2017;45(10):1139-42. DOI:
Nota: Artigo proveniente de trabalho de iniciação científica, intitulado "Modificando a Prática de Desconectar Cateter Venoso Estéril Mediante Intervenção Educativa", do Programa Institucional de Bolsas de Iniciação Científica da Universidade Federal dos Vales do Jequitinhonha e Mucuri.

Recebido em: 09/07/2018

Aprovado em: 20/12/2018

Endereço de correspondência:

Helisamara Mota Guedes

Rodovia MGT 367-Km 583, n 5000-Alto da Jacúba

CEP: 39100-000 - Diamantina/MG - Brasil

E- mail: helisamaraguedes@gmail.com 